\title{
Inhomogeneous Isobaric Poiseuille-Ekman Flow of a Viscous Incompressible Fluid
}

\author{
A. V. Gorshkov ${ }^{1,2, a)}$ and E. Yu. Prosviryakov ${ }^{1,2, b)}$ \\ ${ }^{1}$ Institute of Engineering Science, Ural Branch of the Russian Academy of Sciences, \\ 34 Komsomolskaya St., Ekaterinburg, 620049, Russia \\ ${ }^{2}$ B. N. Yeltsin Ural Federal University, 19 Mira St., Ekaterinburg, 620002, Russia

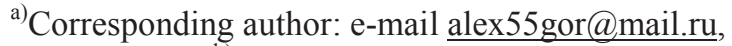 \\ b)evgen_pros@mail.ru
}

\begin{abstract}
An analytical solution describing the stationary isobaric flow of a viscous incompressible slowly rotating fluid in an infinitely extended layer is obtained, with inhomogeneous velocity distribution. The fluid flow is considered at constant pressure, in accordance with the generalization of the classical Ekman flow. The allowance made for velocity gradients can both increase and decrease the number of stagnation points in the fluid layer as compared to the homogeneous Ekman flow. It is shown that the new exact solution allows us to construct new classes of exact solutions of the Navier-Stokes equations describing fluid flow in an inertial coordinate system and in a rotating one.
\end{abstract}

\section{INTRODUCTION}

The classical exact Ekman solution [1] and its modifications [2] are used for the description of large-scale countercurrents in the world's ocean. The structure of the Ekman solution describes the laminar flow of a fluid, which is determined by the balance of inertial and viscous forces. A characteristic feature of the Ekman flow is the assumption of wind uniformity on the free surface of the ocean. In other words, the velocities or tangential stresses specified at the horizontal boundaries of the infinite fluid layer are constant quantities. It is obvious that in reality the distribution of velocities and stresses on the boundaries of a fluid layer is very complex [3]. In this case, when finding exact solutions to the Navier-Stokes equations, it is necessary to take into account the spatial acceleration of the fluid, which is determined by the velocity gradients along the horizontal (longitudinal) coordinates $[4,5]$. If we discuss the solution of the classical Ekman system, the allowance for the longitudinal components of the velocity gradients leads to the solution of the overdetermined system of partial differential equations. Even for linear systems, the analysis of the compatibility of the overdetermined equations is a challenge.

In [6-8], exact solutions of overdetermined nonlinear Navier-Stokes equations describing laminar countercurrents in the absence of a Coriolis force field were given for the first time. Consequently, they are suitable for studying the motion of ocean waters in the equatorial zone. This solution was obtained with the use of a representation of the velocity field linear in the horizontal coordinates [9-16]. This paper considers another limiting case. For the Ekman system, we consider a similar exact solution to the equations of motion of a rotating fluid; the solution has a different physical meaning.

\section{PROBLEM STATEMENT}

The motion of a viscous incompressible fluid in a rotating coordinate system at a constant temperature is described by a dimensionless system of Navier-Stokes equations [4-16]. The equation system written in dimensionless variables with allowance for $V_{z}=0$ has the following form [2, 15-17]:

Mechanics, Resource and Diagnostics of Materials and Structures (MRDMS-2020) AIP Conf. Proc. 2315, 050009-1-050009-6; https://doi.org/10.1063/5.0036899 Published by AIP Publishing. 978-0-7354-4057-9/\$30.00 


$$
\begin{gathered}
\operatorname{Re}\left(V_{x} \frac{\partial V_{x}}{\partial x}+V_{y} \frac{\partial V_{x}}{\partial y}\right)-\frac{\sin \varphi}{E k} V_{y}=-\frac{\operatorname{Re}}{\delta} \frac{\partial P}{\partial x}+\Delta V_{x}, \\
\operatorname{Re}\left(V_{x} \frac{\partial V_{y}}{\partial x}+V_{y} \frac{\partial V_{y}}{\partial y}\right)+\frac{\sin \varphi}{E k} V_{x}=-\frac{\operatorname{Re}}{\delta} \frac{\partial P}{\partial y}+\Delta V_{y}, \\
\operatorname{Re} \frac{\partial P}{\partial z}=\frac{\cos \varphi}{E k} V_{x}, \frac{\partial V_{x}}{\partial x}+\frac{\partial V_{y}}{\partial y}=0 .
\end{gathered}
$$

Here, $V_{x}, V_{y}$ are the dimensionless components of the fluid velocity vector; $P$ is the pressure normalized to twice the specific kinetic energy $\rho V^{2}$; the dimensionless horizontal coordinates $x$ and $y$ are determined by the characteristic length scale $L$, the transverse coordinate $z$ being determined by the thickness $h$ of the fluid layer; $\delta=h / L$ is the ratio of the length scales; $\operatorname{Re}=W L / v$ is the Reynolds number; $\mathrm{Ek}=v /\left(2 L^{2} \Omega\right)$ is the Ekman number, $W$ is the characteristic velocity of the fluid flow, $\Omega$ is the angular velocity of rotation of the fluid; $v$ is the kinematic viscosity of the fluid; $\varphi$ is the latitude of the place; $\Delta=\frac{\partial^{2}}{\partial x^{2}}+\frac{\partial^{2}}{\partial y^{2}}+\frac{1}{\delta^{2}} \frac{\partial^{2}}{\partial z^{2}}$ is the three-dimensional Laplace operator.

Note that the introduction of the horizontal length scale $L$ allows large-scale flows to be described without setting boundary conditions on the vertical (lateral) boundaries of the fluid layer. In other words, an infinitely long band of finite thickness can be used for ocean models since it is always possible to correlate the velocities by selecting the scale $L$ on the side boundaries. In this case, there will be a finite velocity value on the side boundaries. The choice of an infinite layer is due to the only fact that, when large-scale flows are studied, the study of the wind dependence of currents comes to the fore, in the assumption that the coast is far away. In other words, the motions of a rotating viscous incompressible fluid are studied at small Froude numbers near the axis of rotation [2].

\section{EXACT SOLUTION}

The exact solution of the overdetermined system of equations (1) is calculated in the following form [6-11]:

$$
V_{X}=U_{0}+X U_{1}+Y U_{2}, V_{Y}=V_{0}+X V_{1}+Y V_{2}
$$

To calculate the unknown functions $U_{i}, V_{i}, i=\overline{0,2}$ depending on the transverse coordinate $z$, substitute the velocity field (2) into (1) and obtain the following system of ordinary differential equations:

$$
\begin{gathered}
\frac{d^{2} U_{1}}{d z^{2}}=-2 \delta^{2} k^{2} V_{1}, \frac{d^{2} V_{1}}{d z^{2}}=2 \delta^{2} k^{2} U_{1}, \\
\frac{d^{2} U_{2}}{d z^{2}}=-2 \delta^{2} k^{2} V_{2}, \frac{d^{2} V_{2}}{d z^{2}}=2 \delta^{2} k^{2} U_{2}, \\
\frac{d^{2} U_{0}}{d z^{2}}=-2 \delta^{2} k^{2} V_{0}, \frac{d^{2} V_{0}}{d Z^{2}}=2 \delta^{2} k^{2} U_{0}, \\
U_{1}+V_{2}=0 .
\end{gathered}
$$

Here, the dimensionless dispersion number is denoted by $k=1 / \sqrt{2 E k}$. Note that, if we consider only homogeneous terms $U_{0}, V_{0}$ in the representation of velocities (2), we obtain the classical Ekman flow.

We analyze the solvability of the overdetermined system (3)-(6), which consists of three subsystems of the same structure (3)-(5) and the incompressibility condition (6). It follows from the equality (6) that $U_{1}=-V_{2}$. Substituting this relationship of the horizontal components of the velocity gradient into (4), we obtain 


$$
\frac{d^{2} U_{2}}{d z^{2}}=2 \delta^{2} k^{2} U_{1}, \frac{d^{2} U_{1}}{d z^{2}}=-2 \delta^{2} k^{2} U_{2}
$$

Analyzing the subsystems (3) and (7), we state that the following equality is true:

$$
V_{1}=U_{2}
$$

This equality is the compatibility condition of the overdetermined system (3)-(6) and, as a consequence, of the Navier-Stokes equations (1) within the class of exact solutions (2).

Substituting (8) into (3), we arrive at the following system of fourth-order ordinary differential equations:

$$
\frac{d^{2} U_{1}}{d z^{2}}=-2 \delta^{2} k^{2} U_{2}, \frac{d^{2} U_{2}}{d z^{2}}=2 \delta^{2} k^{2} U_{1}
$$

Obviously, equations (9) describe the behavior of the velocity gradients, and their structure coincides with system (5). Consequently, the equation systems (5) and (9) have an identical general solution expressed through quasipolynomials $[15,16]$.

The fluid flow is considered in an infinite horizontal layer bounded from below by the plane $z=0$ (bottom), where the no-slip conditions are met,

$$
U_{0}(0)=0, V_{0}(0)=0, U_{1}(0)=0, U_{2}(0)=0,
$$

and the following velocities are set on the upper boundary $z=1$ :

$$
U_{0}(1)=W \cos \varphi, V_{0}(1)=W \sin \varphi, U_{1}(1)=A, U_{2}(1)=B
$$

With regard for the boundary conditions (10) and (11), the solution of systems (5) and (9) has the following form:

$$
\begin{gathered}
U_{0}=\frac{W}{1+e^{4 k \delta}-2 e^{2 k \delta} \cos (2 k \delta)}\left[e^{k \delta(3+z)} \cos (k \delta(1-z)-\varphi)-\right. \\
\left.-e^{k \delta(3-z)} \cos (k \delta(1+z)-\varphi)+e^{k \delta(1-z)} \cos (k \delta(1-z)+\varphi)-e^{k \delta(1+z)} \cos (k \delta(1+z)+\varphi)\right] \\
V_{0}=\frac{W}{1+e^{4 k \delta}-2 e^{2 k \delta} \cos (2 k \delta)}\left[-e^{k \delta(3+z)} \sin (k \delta(1-z)-\varphi)+\right. \\
\left.+e^{k \delta(3-Z)} \sin (k \delta(1+z)-\varphi)+e^{k \delta(1-z)} \sin (k \delta(1-z)+\varphi)-e^{k \delta(1-z)} \sin (k \delta(1+z)+\varphi)\right] \\
U_{1}=A \frac{\left(e^{k \delta(1-z)}+e^{k \delta(z+3)}\right) \cos (k \delta(1-z))-\left(e^{k \delta(3-z)}+e^{k \delta(1+z)}\right) \cos (k \delta(1+z))}{1+e^{4 k \delta}-2 e^{2 k \delta} \cos (2 k \delta)}+ \\
+B \frac{\left(e^{k \delta(z-1)}-e^{-k \delta(3+z)}\right) \sin (k \delta(1-z))-\left(e^{-k \delta(1+z)}-e^{k \delta(z-3)}\right) \sin (k \delta(1+z))}{1+e^{4 k \delta}-2 e^{2 k \delta} \cos (2 k \delta)} \\
U_{2}=B \frac{\left(e^{k \delta(1-z)}+e^{k \delta(z-1)}\right) \cos (k \delta(1-z))-\left(e^{-k \delta(z+1)}+e^{k \delta(z-3)}\right) \cos (k \delta(1+z))}{1+e^{4 k \delta}-2 e^{2 k \delta} \cos (2 k \delta)}+ \\
+A \frac{\left(e^{k \delta(1-z)}-e^{k \delta(3+z)}\right) \sin (k \delta(1-z))+\left(e^{k \delta(3-z)}-e^{k \delta(1+z)}\right) \sin (k \delta(1+z))}{1+e^{4 k \delta}-2 e^{2 k \delta} \cos (2 k \delta)}
\end{gathered}
$$


The solution represented by (12), (13) contains exponential terms that tend to infinity at infinitely large values of $z$. When considering fluid flows in a layer of finite thickness, it is necessary to take into consideration all the terms determining velocities and velocity gradient components in (2).

To study the properties of the flow, we present its asymptotics at large values of the product $k \delta$ :

$$
\begin{gathered}
U_{1}(z)=A e^{k \delta(z-1)} \cos (k \delta(z-1))-B e^{k \delta(z-1)} \sin (k \delta(z-1)), \\
U_{2}(z)=A e^{k \delta(z-1)} \sin (k \delta(z-1))+B e^{k \delta(z-1)} \cos (k \delta(z-1)), \\
U_{0}(z)=W e^{k \delta(z-1)} \cos (k \delta(1-z)-\varphi), V_{0}(z)=-W e^{k \delta \delta(z-1)} \sin (k \delta(1-z)-\varphi) .
\end{gathered}
$$

It is obvious that the exact solution (12), (13) is a helical screw line unwinding according to the exponential law with the index $k \delta(z-1)$, Fig. 1.

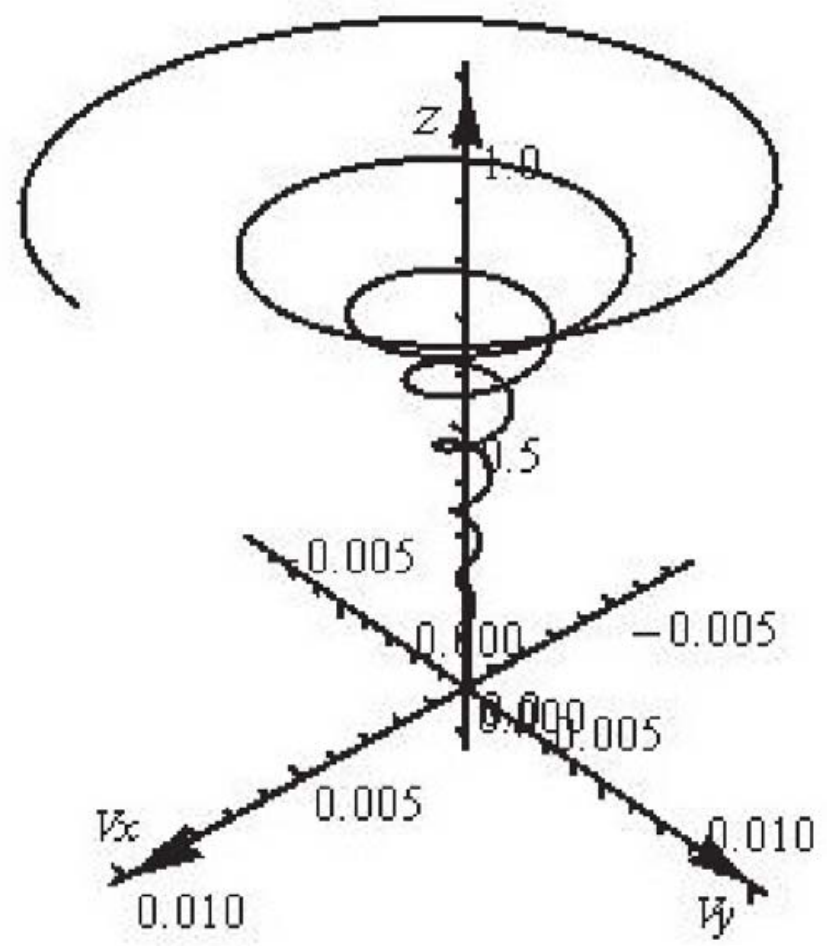

FIGURE 1. The classical Ekman solution

Solution (12) determines the existence of counterflows in the fluid, which were first described by Ekman for a rotating fluid at zero velocity gradients [1]. In this case, the structure of the fluid flow is shown in Fig. 2. Due to the complete accounting of the exponential terms, the structure differs significantly from the classical Ekman solution (Fig. 2). The "total" velocity is $V_{x}=U_{0}+x U_{1}+y U_{2}$ and $V_{y}=V_{0}+x V_{1}+y V_{2}$ when gradients are taken into account (red curve) and $V_{x}=U_{0}, V_{y}=V_{0}$ without a gradient (black curve). 


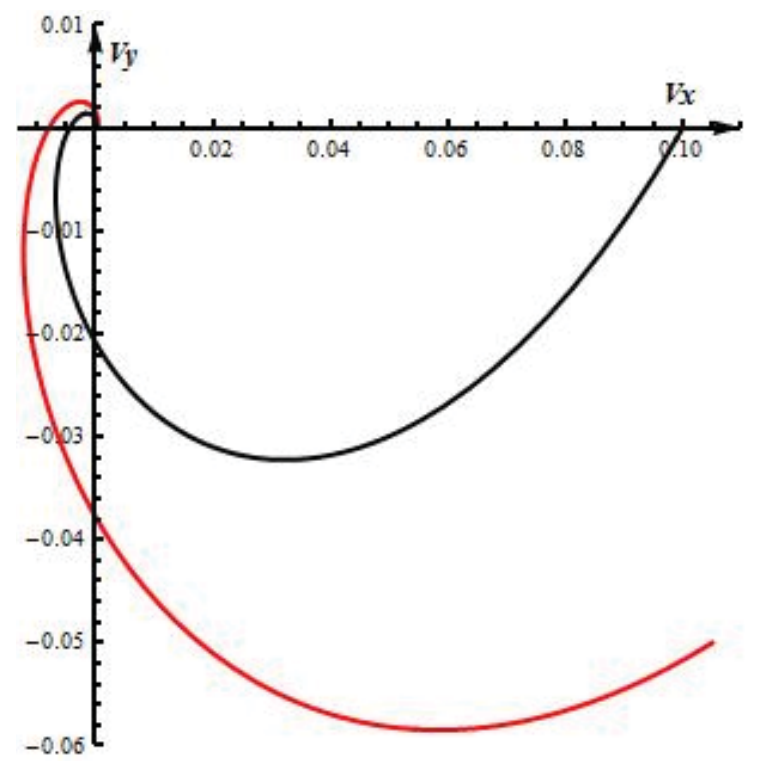

FIGURE 2. Hodographs of the velocities $V_{x}, V_{y}$ calculated for the parameter values $W=0.1, \varphi=0, A=0.1, B=0.01$, and $k=70$; at the point $x=0, y=0.5$

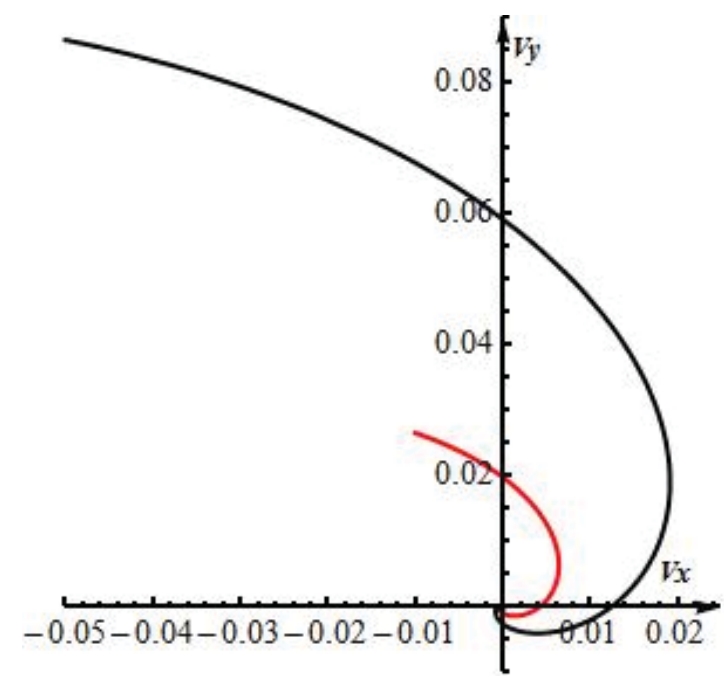

FIGURE 3. Hodographs of the velocities $V_{x}, V_{y}$ calculated for the parameter values $W=0.1, \varphi=2 \pi / 3, A=0.1, B=0.01$, and $k=70$; at the point $x=-0.1, y=0.5$

If the velocities and their gradients are nonzero at the boundary between the atmosphere and the ocean, the cross effects of their interaction can both increase and decrease the number of stagnation points (Fig. 3).

Finally, note that the exact solution (2) allows us to obtain a new class of exact solutions of the Navier-Stokes equations in an inertial coordinate system. To do this, we recall that the exact Ekman solution can be found with the use of the Navier-Stokes equations if the velocities are represented as

$$
V_{x}=U_{0}(z)-f y, V_{y}=V_{0}(z)+f x .
$$

Using a similar reasoning, valid for the Lin-Sidorov-Aristov class of exact solutions, we obtain a new family of exact solutions for the Navier-Stokes equations to describe three-dimensional laminar flows of a viscous incompressible fluid,

$$
\begin{gathered}
V_{x}=U_{0}+x U_{1}+y U_{2}+U_{11} \frac{x^{2}}{2}+U_{12} x y+U_{22} \frac{y^{2}}{2}, \\
V_{y}=V_{0}+x V_{1}+y V_{2}+V_{11} \frac{x^{2}}{2}+V_{12} x y+V_{22} \frac{y^{2}}{2} .
\end{gathered}
$$

In addition, for rotating fluids, solution (2) allows the following generalization:

$$
\begin{aligned}
& V_{x}=U_{0}(z)+\sum_{m=1}^{n} \frac{1}{m !} \sum_{k=0}^{m} C_{m}^{k} U_{k, m-k}(z) x^{k} y^{m-k}, \\
& V_{y}=V_{0}(z)+\sum_{m=1}^{n} \frac{1}{m !} \sum_{k=0}^{m} C_{m}^{k} V_{k, m-k}(z) x^{k} y^{m-k} .
\end{aligned}
$$

Here, $C_{n}^{k}$ is the number of combinations without repetitions. The choice of the number of terms is dictated by the boundary conditions of the problem. It is obvious that the obtained new classes of exact solutions are valid for non-stationary flows if the functions defining the structure of the solution depend not only on the transverse coordinate, but also on time. 


\section{CONCLUSION}

An analytical solution describing the slow Ekman flow has been obtained. Its asymptotic approximation has been constructed. The difference between the gradient-based flow and the classical Ekman flow has been demonstrated. The possibility of the appearance of stagnation points has been shown.

\section{REFERENCES}

1. V. W. Ekman, Arkiv för Matematik, Astronomi, och Fysik. Band 2. 11 1-53 (1905).

2. A. I. Felzenbaum, Theoretical Bases and Methods for Calculating Steady Sea Currents (AN USSR, Moscow, 1960).

3. J. Pedlosky, Geophysical Fluid Dynamic (Springer-Verlag, New York Inc., 1982).

4. G. K. Korotaev, E. N. Mikhaylova, and N. B. Shapiro, Theory of Equatorial Countercurrents in the World's Ocean (Naukova Dumka, Kiev, 1986).

5. V. N. Zyryanov, Theory of Steady Ocean Currents (Gidrometeoizdat, Leningrad, 1985).

6. S. N. Aristov and E. Yu. Prosviryakov, Nonlinear Dynamics 10 (2), 177-182 (2014).

7. S. N. Aristov and E. Yu. Prosviryakov, Nonlinear Dynamics 9 (3), 3-9 (2013).

8. L. Kh. Ingel and S. N. Aristov, Institute of Experimental Meteorology 27(162), 142-157 (1996).

9. S. N. Aristov and E. Yu. Prosviryakov, Fluid Dynamics 2, 25-31 (2016).

10. S. N. Aristov and E. Yu. Prosviryakov, Theoretical Foundations of Chemical Engineering 50(3) 294-301 (2016).

11. E. Yu. Prosviryakov, Theoretical Foundations of Chemical Engineering 53 (1), 112-120 (2019).

12. S. N. Aristov and K. G. Shvarts, Vortical Flows of Advective Nature in a Rotating Fluid Layer (PSU, Perm, 2006).

13. S. N. Aristov and K. G. Shvarts, Vortical Flows in Thin Fluid Layers (Vyatka State Univ., Kirov, 2011).

14. S. N. Aristov and K. G. Shvarts, Journal of Applied Mechanics and Technical Physics 1, 216-223, 2016).

15. A. V. Gorshkov, E. Yu. Prosviryakov, AIP Conference Proceedings 1915, 040020 (2017).

16. A. V. Gorshkov and E. Yu. Prosviryakov, Izvestiya. Atmospheric and Oceanic Physics 54 (2), 189-195 (2018).

17. A. V. Gorshkov and E. Yu. Prosviryakov, AIP Conference Proceedings 1915, 040019 (2017).

18. A. V. Gorshkov and E. Yu. Prosviryakov, AIP Conference Proceedings 2053, 040030 (2018).

19. A. V. Gorshkov and E. Yu. Prosviryakov AIP Conference Proceedings 2053, 040029 (2018). 